Mark J. Peters

Rachel S. Agbeko

\section{Optimism and no longer foolishness? Haematology/oncology and the PICU}

Received: 26 August 2014

Accepted: 28 August 2014

Published online: 18 September 2014

(C) The Author(s) 2014. This article is published with open access at Springerlink.com

\section{J. Peters (『)}

Respiratory Critical Care and Anaesthesia Unit, Institute of Child Health, University College London, London WC1N 1EH, UK e-mail: mark.peters@ucl.ac.uk

\section{J. Peters}

Paediatric Intensive Care Unit, Great Ormond Street Hospital for Children NHS Trust, London WC1N 3JH, UK

\section{R. S. Agbeko}

Paediatric Intensive Care Unit, Great North Children's Hospital, The Newcastle Hospitals, NHS Foundation Trust, Newcastle upon Tyne NE1 4LP, UK

\section{R. S. Agbeko}

Institute of Cellular Medicine, Newcastle University, Newcastle upon Tyne NE2 4HH, UK

Theodore Roosevelt [1] said: "Optimism is a good characteristic but if carried to excess becomes foolishness." Something similar-but less eloquent—might often have been said by a paediatric intensivist to a haematology/ oncology colleague. Or worse: a senior colleague once asked: "Why do we put nails in coffin lids?" Before the punchline: "To stop the oncologists giving one more round of chemo." This is clearly now (and was then) an absurd exaggeration. But perhaps what lies behind this tension is captured in Roosevelt's [1] next line: "We are prone to speak of the resources of this country as inexhaustible. This is not so." Neither are paediatric intensive care resources inexhaustible. Therefore, intensivists are used to balancing costs and benefits of aggressive or palliative treatment. We, consciously or unconsciously, ration our limited resources [2]. But could bad outcomes, such as those perceived for children with cancer, become a self-fulfilling prophecy with out-of-date risks informing plans to withdraw or withhold treatments? How do we avoid circular arguments wherein our perceptions of poor prognosis may contribute to poor outcomes in the plans we make with families or the intensity of the support we offer?

The answer is in large, systematically collected data sets. Many paediatric intensivists are aware that the paediatric index of mortality (PIM score) $[3,4]-$ now in its third full iteration (PIM3) [5] — describes a much higher PICU mortality for the same admission physiology amongst haematology/oncology cases than the general PICU population. The terms 'leukaemia/lymphoma outside of first induction' or 'bone marrow transplant recipient' both represent 'very high-risk' diagnoses. In 53,112 recent cases, either of these fields carries a fivefold greater risk of death with equivalent severity of organ failure at admission. This is the same tariff as a cardiac arrest preceding PICU admission [5].

This dismay-inducing comparison needs to be seen in the light of the knowledge of the haematologist/oncologist that many children with cancer $(38 \%$ at some point in their illness) will be admitted at to a PICU [6]. And they know that their meticulous cancer registry data show impressive and improving results. Five-year survival for a child with cancer is now at $83 \%$ [7]. So for a haematologist/oncologist, PICU is a frequent stopover on a pathway to cure.

To try to understand and reconcile these differing assessments of risk and benefit, we must be precise in our descriptions of patients. Zinter et al. [8] have provided a description of outcomes for 10,365 paediatric cancer emergency admissions out of 246,346 admissions to the US virtual PICU systems database. The overall mortality was only $6.8 \%$. We should take note of this number. It is 
lower than most series quote for previously healthy children with community-acquired sepsis or acute respiratory distress syndrome (ARDS) on PICU [9-12]. The observed relative risk of PICU death is still highly significant at 2.9 (95\% CI 2.7-3.1), but the truth is that this reflects the overall improvement in PICU outcomes more than cancer lagging behind. Indeed, if we compare the predicted risk of mortality for a child with sepsis-induced multiple organ dysfunction syndrome (MODS) or ARDS, with and without a high risk category of 'leukaemia/ lymphoma outside of first induction' through each of the four published versions of the PIM scores since 1997, we see that the outcomes for cancer cases have been modelled as improving much faster than those of healthy children (Fig. 1). These results challenge us to understand the underlying mechanisms and to further refine and reflect on our practise. Similar trends have been observed in adult haematology and cancer [13]. It is unclear if the general improvements in ICU (e.g. early recognition and treatment of sepsis, or low tidal volume ventilation in ARDS) have been disproportionately effective in haematology/oncology cases. This is a credible explanation considering their higher baseline risk. Or does this arise from a specific therapy relevant to haematology/oncology? Certainly tumour lysis syndrome therapies are now highly effective. The change in mortality for adult cancer patients with ARDS is striking: $89 \%$ mortality 1990-1995 vs. 52\% 2007-2011 [14]. This raises the possibility of a greater gain from more widespread use of non-invasive ventilation in this population $[15,16]$. This hypothesis is currently under investigation in children
("SCARF" trial ISRCTN82853500) and adults ("IVNICTUS" trial NCT01915719).

There are more tantalising clues to the underlying mechanisms in Zinter et al.'s paper. Acute myeloid leukaemia was independently associated with high riskconsistent with recent work [17]. This might reflect the more immunosuppressive regimens. Certainly infection remains an independent risk factor. However, we know that the outcomes for neutropenic fever are now excellent (case fatality rate $0.7 \%, 95 \%$ CI $0.2-2.1$ ) [18]. So perhaps these results also reflect toxicities of chemotherapy that matter more in the context of critical illness (e.g. cardiac toxicity of anthracyclines).

But we do not need speculation or further work, however, to recognise at least one important result in this paper: extracorporeal membrane oxygenation (ECMO) in haematology/oncology patients was associated with a $50 \%$ survival (18/36 cases). This contrasts with the still dismal results for ECMO post haemopoietic stem cell transplantation [19]. Perhaps this is one directly relevant and clear distinction between optimism and foolishness?

Most in-hospital deaths occur following withdrawal of intensive care. This clinical decision-making process is now better informed by an increased understanding of epidemiology as presented by Zinter. Further enrichment is now required, describing patient pathways, mode of death, and underlying pathophysiology. To do this we need increased dialogue and sharing of data between haematology/oncology and PICU. This should not be difficult; after all we are two of the most successful, bestresourced and high profile paediatric specialties. Both

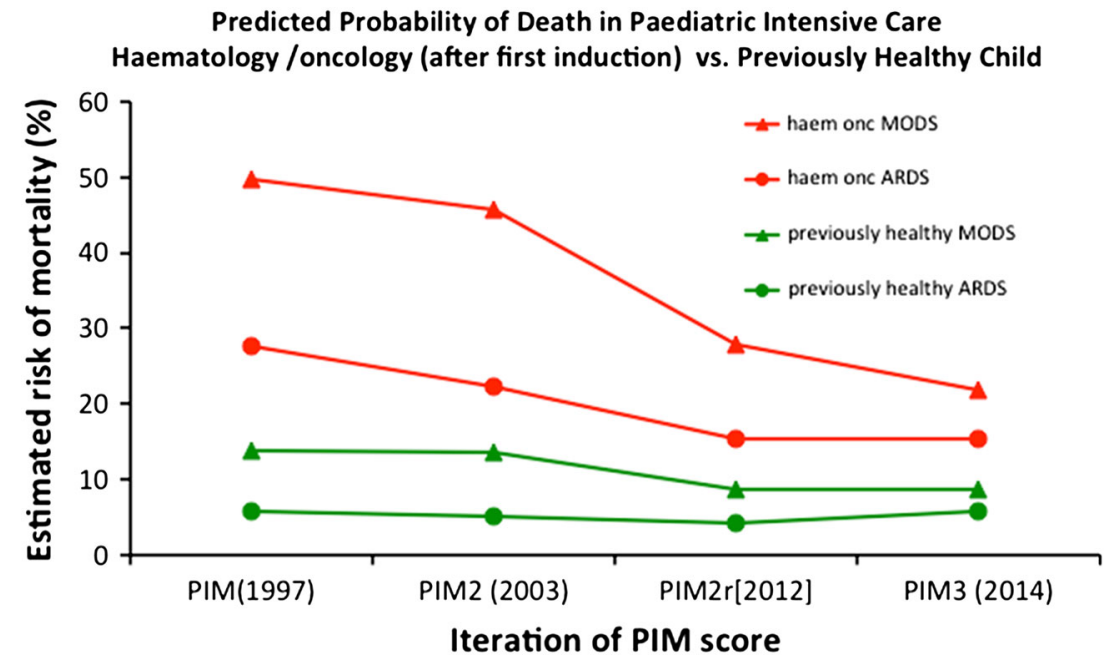

Fig. 1 Predicted probability of death in the paediatric intensive care unit from successive iterations of the paediatric index of mortality score (PIM) with acute respiratory distress syndrome (ARDS) or multiple organ dysfunction syndrome (MODS). Examples with equivalent admission physiology, with and without the high-risk diagnosis of 'leukaemia/lymphoma outside first

induction' are shown. The absolute (ARR) and relative risk reductions (RRR) for haematology/oncology cases are greater than for the non-high-risk cases in this period (PIM vs. PIM3, ARDShigh risk: ARR $12.2 \%$, RRR $44.2 \%$; non-high risk: ARR $0 \%$, RRR $0 \%$. MODS-high risk: ARR $28 \%$, RRR 56.2 \%; non-high risk: ARR $5.1 \%$, RRR $37 \%$ ) 
have developed rapidly in the last 40 years and constitute a large proportion of the in-patient facilities, staff and resources in tertiary children's hospitals. We share a preoccupation with infection and the immune response. Most importantly both specialities have seen dramatic and continuous improvements in outcome. Survival is now the expected outcome for a cancer diagnosis or an episode of critical illness in a child. Each has refined techniques (mechanical ventilation or bone marrow transplant conditioning) that have resulted in dramatic reductions in iatrogenic harm and improved survival. Each has huge (perhaps the largest?) impacts on child mortality. We frequently share patients. Work like that of Zinter and colleagues is essential to optimise this collaboration.
Acknowledgments This work was undertaken at Great Ormond Street Hospital/UCL Institute of Child Health, which received a proportion of funding from the Department of Health's NIHR Biomedical Research Centre's funding scheme.

Conflicts of interest No conflicts of interest to declare.

Open Access This article is distributed under the terms of the Creative Commons Attribution Noncommercial License which permits any noncommercial use, distribution, and reproduction in any medium, provided the original author(s) and the source are credited.

\section{References}

1. Roosevelt T (1907) Seventh annual message to Congress.

http://www.presidency.ucsb.edu/ws/ ?pid=29548. Accessed 15 Sept 2014

2. Truog RD, Brock DW, Cook DJ et al (2006) Rationing in the intensive care unit. Crit Care Med 34:958-963. doi: $10.1097 /$

01.CCM.0000206116.10417.D9

3. Shann F, Pearson G, Slater A, Wilkinson K (1997) Paediatric index of mortality (PIM): a mortality prediction model for children in intensive care. Intensive Care Med 23:201-207

4. Slater A, Shann F, Pearson G, Paediatric Index of Mortality (PIM) Study Group (2003) PIM2: a revised version of the paediatric index of mortality. Intensive Care Med 29:278-285. doi: 10.1007/s00134-002-1601-2

5. Straney L, Clements A, Parslow RC et al (2013) Paediatric index of mortality 3: an updated model for predicting mortality in pediatric intensive care. Pediatr Crit Care Med 1-9:673-681. doi: 10.1097/PCC.0b013e31829760cf

6. Rosenman MB, Vik T, Hui SL, Breitfeld PP (2005) Hospital resource utilization in childhood cancer. J Pediatr Hematol Oncol 27:295-300

7. National Cancer Institute (2014) Surveillance, epidemiology, and end results (SEER) program research data (1973-2011)

8. Zinter MS, DuBois SG, Spicer A et al (2014) Pediatric cancer type predicts infection rate, need for critical care intervention, and mortality in the pediatric intensive care unit. Intensive Care Med. doi: 10.1007/s00134-014-3389-2
9. Inwald DP, Tasker RC, Peters MJ et al (2009) Emergency management of children with severe sepsis in the United Kingdom: the results of the Paediatric Intensive Care Society sepsis audit. Arch Dis Child 94:348-353. doi: 10.1136/adc.2008.153064

10. De Luca D, Piastra M, Chidini G et al (2013) The use of the Berlin definition for acute respiratory distress syndrome during infancy and early childhood: multicenter evaluation and expert consensus. Intensive Care Med 39:2083-2091. doi: 10.1007/s00134-013-3110-x

11. Deep A, Goonasekera CDA, Wang Y, Brierley J (2013) Evolution of haemodynamics and outcome of fluidrefractory septic shock in children. Intensive Care Med 39:1602-1609. doi: 10.1007/s00134-013-3003-z

12. Hartman ME, Linde-Zwirble WT, Angus DC, Watson RS (2013) Trends in the epidemiology of pediatric severe sepsis. Pediatr Crit Care Med 1:686-693. doi: 10.1097/PCC.0b013e3182917fad

13. van Vliet $M$, Verburg IWM, van den Boogaard $\mathrm{M}$ et al (2014) Trends in admission prevalence, illness severity and survival of haematological patients treated in Dutch intensive care units. Intensive Care Med. doi: 10.1007/s00134-014-3373-x

14. Azoulay E, Lemiale V, Mokart D et al (2014) Acute respiratory distress syndrome in patients with malignancies. Intensive Care Med 40:1106-1114. doi: 10.1007/s00134-014-3354-0
15. Hilbert G, Gruson D, Vargas F et al (2001) Noninvasive ventilation in immunosuppressed patients with pulmonary infiltrates, fever, and acute respiratory failure. $\mathrm{N}$ Engl J Med 344:481-487. doi: 10.1056/NEJM200102153440703

16. Depuydt PO, Soares M (2014) Cancer patients with ARDS: survival gains and unanswered questions. Intensive Care Med 40:1168-1170. doi: 10.1007/s00134-014-3394-5

17. Maude SL, Fitzgerald JC, Fisher BT et al (2014) Outcome of pediatric acute myeloid leukemia patients receiving intensive care in the United States. Pediatr Crit Care Med 15:112-120. doi: 10.1097/PCC.0000000000000042

18. Lüthi F, Leibundgut K, Niggli FK et al (2012) Serious medical complications in children with cancer and fever in chemotherapy-induced neutropenia: results of the prospective multicenter SPOG 2003 FN study. Pediatr Blood Cancer 59:90-95. doi: $10.1002 /$ pbc. 23277

19. Di Nardo M, Locatelli F, Palmer K et al (2014) Extracorporeal membrane oxygenation in pediatric recipients of hematopoietic stem cell transplantation: an updated analysis of the Extracorporeal Life Support Organization experience. Intensive Care Med 40:754-756. doi: 10.1007/s00134-014-3240-9 